\title{
RAMS/LCC ANFORDERUNGEN IM IRIS STANDARD - HERAUSFORDERUNG FÜR UNTERNEHMEN AUS DER SCHIENENFAHRZEUGINDUSTRIE
}

Fatih ÖZTÜRK'

Vienna University of Technology, Vienna, Austria

\section{ABSTRACT}

To ensure and enable a system to be used to determine quality and distress in the rail sector, a standard was created for the companies. Meanwhile, there are 13 companies having the IRIS certificate which started to be used in 2006. And, the number is growing day by day. In many ways, it is difficult to meet the wishes of the clients and the service by the producers. This is probably due to the lack of knowledge and experience of both clients and manufacturers. So we can say that now there is a standard to meet the wishes of clients and the service of the manufacturers. Most probably, the hardest thing to give a good service is in the area of the RAMS/LCC management, which is overwhelmed by only a few companies. This is going to be discussed in greater details to give an accurate point of view from the companies' perspective.

\section{JEL CLASSIFICATION \& KEYWORDS}

- L15 | L23 | M11 | RAILWAY - IRIS - QUALITY MANAGEMENT - RAILWAY INDUSTRY — RAMS/LCC

\section{ZUSAMMENFASSUNG}

Um eine Teilhabe am internationalen Markt zu garantieren bzw. zu ermöglichen ist auch für Unternehmen aus dem Schienensektor ein funktionierendes Qualitätsmanagementsystem von Nöten. Zuspruch fand hierfür vor allem der Branchenstandard IRIS, welches im Jahre 2006 erstmals in Anwendung kam und mittlerweile auch 13 Unternehmen aus der Türkei ein Zertifikat besitzen. Der Trend ist steigend. In vielerlei Hinsicht scheint dieser Standard den Wünschen der Unternehmungen gerecht $\mathrm{zu}$ werden, doch auch Unmutstöne sind von einigen zuhören, die besonders auf die geringen Kenntnisse und Erfahrung der einzelnen Klienten zurückzuführen sind. Anzuführen ist auch die Tatsache, dass IRIS ein erst recht neuer Standard ist und deshalb auch je nach Schwierigkeiten und Wünschen des Klientels nach oben hin Aufbaufähig ist. Schwierigkeiten seitens der Befragten Unternehmen sind vor allem im Bereich des RAMS/LCC Management zu hören, die einige Firmen überfordern. In dieser Arbeit wird auch genauer auf dieses eingegangen und genauer aus der Sich der Unternehmen betrachtet.

\section{Schienenfahrzeugindustrie in der Türkei}

Der Schienensektor in der Türkei wurde lange Zeit vernachlässigt und erst in den letzten 10 Jahren hat es wieder an Bedeutung zugewonnen. Enorme Investitionen des Staates für Privatunternehmen in diesem Sektor, Ausbau des Schienennetzes sowie Bau, von Hochgeschwindigkeitszügen und Straßenbahnen sind einige diese Innovationen. Der rapide Zuwachs dieses Sektors in der Türkei, war auch ein Anreiz für bestehende Unternehmen unter anderem aus der Automobilindustrie sich in diesem Sektor zu betätigen. Um jedoch effizient am Markt teilhaben zu können, ist ein funktionierendes international anerkanntes Branchenstandard für das

\footnotetext{
I fatihozturk79@gmail.com
}

www.journals.cz
Unternehmen essentiell. Weshalb auch immer mehr Unternehmen sich für IRIS entscheiden. Untersucht wurde in diesem Kontext, jene Schwierigkeiten die mit der Implementierung von IRIS für türksiche Unternehmen einhergeht. Dabei Kristallisierten sich vor allem im Bereich RAMS /LCC (Zuverlässigkeit, Verfügbarkeit, Instandhaltbarkeit, Sicherheit /Lebenszykluskosten) Management unmutstöne.

Es herrscht Unklarheit über die Funktionsweise des Systems, sowie deren Einführung. Nachdem auch einige Unternehmen erst neu im Sektor sind, fehlen einigen das nötige Handwerk, die Kenntnisse sowie Erfahrungen. Wichtig anzuführen ist auch, dass die Produktionen in den Unternehmungen keine eigen Entwicklung sind und in vielen Fällen ein umfangreiches RAMS/LCC auch nicht von nöten ist.

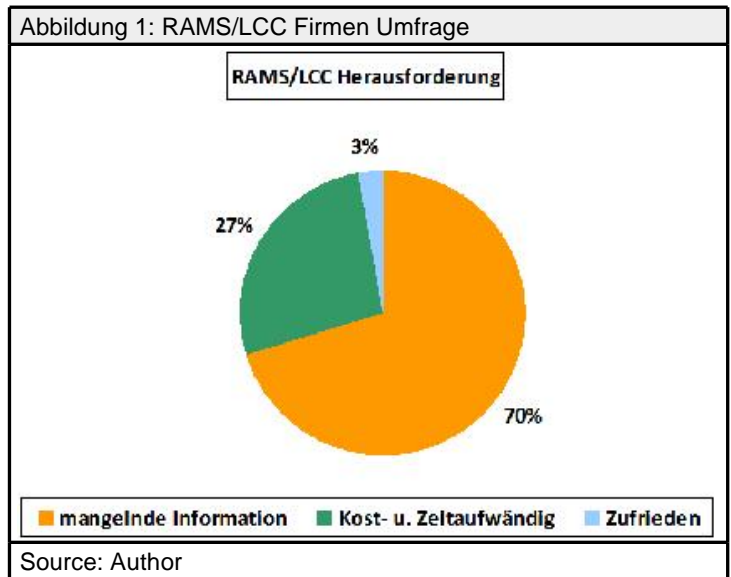

Analysiert wurden türkische Unternehmen, die im Besitz eines IRIS Zertifikates sind. In den geführten Experteninterviews bekundeten $70 \%$ der Befragten Unzufriedenheit, die aus mangelnden Informationen zu diesem Punkt resultieren. Denn obwohl LCC in den "Knock-Out Kriterien" wiederzufinden ist, scheint es für einige Firmen unschlüssig zu sein und eine detaillierte Hilfestellung von Nöten. Aufgrund der unterschiedlichen Produktionszeiten Projektgrößen und Produktion von unterschiedlichen Komponenten sind auch $27 \%$ der Befragten bezüglich der Notwendigkeit eines RAMS oder LCC im Unklaren. Denn RAMS/LCC wird als Kost- und Zeitaufwändig betrachtet und bei kleinen Projekten als nicht notwendig erachtet. Nur $3 \%$ der Befragten hingegen führen keine Schwierigkeiten an. Dies ist aber auch wieder darauf zurück zu führen, dass zum einen Erfahrungen in diesem Bereich vorhanden sind und zum anderen auch Unternehmen zum Thema eigene Workshops absolvieren.

\section{RAMS /LCC in der Schienenfahrzeugindustrie}

Die Zulieferer Firmen entwickeln und produzieren unterschiedliche Eisenbahnkomponente die extrem robust 
und langlebig sein müssen. Denn es wird von Schienenenfahrzeugen eine doch längere Einsatzfähigkeit erwartet. Lebeszyklen von mindestens 10 Jahren und mehr sind gefordert. Wenn die einzelnen Komponente wie auch die Elektronik doch einmal irreparable Schaden aufweisen sollte, sollten identische Produkte lieferbar sein. Daher ist insbesondere für die Schienenfahrzeugindustrie ein geregeltes Lebenszyklusmanagement von großer Bedeutung.

Deshalb zählt zu den Besonderheiten von IRIS die detailtreue Dokumentation. Verfahren und Prozesse werden mittels Kennzahlen erfasst, um Leistungen zu messen, analysieren und $\mathrm{zu}$ verbessern. $\mathrm{Zu}$ den wichtigsten Verfahren zählen in diesem Kontext RAMS / LCC. Das RAMS-Management soll sicher stellen, das Risikoanalysen durfgeführt, Gefährdungsraten ermittelt, detaillierte Prüfungen gemacht und Sicherheitsnachweis erstellt wird (Früh et al. 2009, 746f). Konsequente Dokumentation und Duchführung der erforderlichen Maßnahmen garantieren hochwertiger Produkte und Minimierung von Ausfällen und Gefahrensituationen. Denn durch eine RAMS-Analyse weden Kennzahlen gewonnen, die das Betriebsverhalten des gesamten Systems und der einzelnen Komponenten beschreiben. RAMS wiedmet sich vorwiegend den technischen und organisatorischen Bereich und durch LCC (LebenszyklusKosten) erhält man Aufschluss über die eigentliche Wirtschaftlichkeit (ebd.).

Die Unternehmen erkennen auch, das bei großen Bahnaussreibungen, Kernelemente wie RAMS/LCC zunehmend an Bedeutung gewinnen. Umso wichtiger ist es daher auch für die Zuliefer sich Fachwissen anzueigenen und schon in der Entwicklungsphase mit RAMS /LCC zu arbeiten.

Es zeigt sich jedoch, das genau bei diesem Mangement einige Firmen in der Türkei schwierigkeiten haben. Sie begklagen unter anderem mangelnde Informationen und wünschen sich eine für ihren Betrieb und Produktionsspezifische Hilfestellung.

Im RAMS Mangagement sind Produkt und Prozess eng miteinander verbunden. Für den RAMS-Prozess sind die Ermittlung und Analysen von Kennzahlen wie, zwischen den beteiligten Personen Betreiber, Anlagenhersteller, Zulieferant uvm von enormer Wichtigkeit.

Während den Gesprächen mit den Experten aus den Unternehmen konnte ein kritischer Umgang mit Produktrisiken festgestellt werden, in dem die Betriebe versuchen sich diesen Anforderungen zu stellen. Erschwer werden die Situation und die Ermittlung der Kennzahlen, durch die noch geringen Felddaten die für die RAMS/LCC Ermittlung zur Verfügung stehen. Weiteres können auch einzelne Komponente in den Fahrzeugen nicht geteste werden. Es werden daher Werte von Machbarkeitsstudien und Test ermittelt, die von speziell dafür konstruierten Prüfständen ermittelt werden.

\section{Funktionsweise von RAMS}

Die Definition von RAMS findet sich in der CENELEC Norm EN 50126. In dem RAMS für Reliability Zuverlässigkeit, Availibility - Verfügbarkeit, Maintainability - Instandhaltbarkeit Safety - Sicherheit steht. Sie ist ein Prozess oder eine Methodik, die mithelfen soll, Fehler schon in der Planungsphase von Projekten zu verhindern.

Die Ziele der Sicherheit und der Verfügbarkeit im Betrieb lassen sich nur dann verwirklichen, wenn Zuverlässigkeits und Instandhaltbarkeitsanforderungen ständig erfüllt und

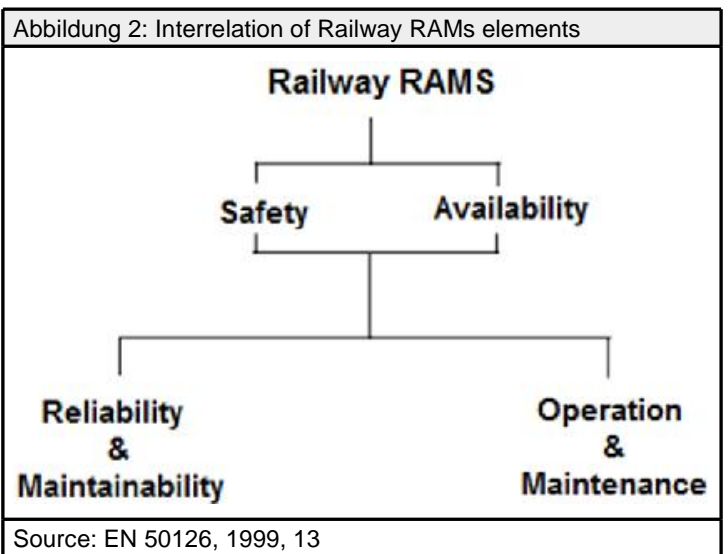

die laufenden langfristigen Instandhaltungsarbeiten sowie das betriebliche Umfeld überwacht werden (Früh et.al, 2009:744f)

Ermittlung der Verfügbarkeit in der RAMS basieren auf die Kenntnisse der (ebd.)

a) Vorkenntnisse, Erfahrungswerte der Zuverlässigkeit aus den Systemausfallraten,

b) Gefährdungsanalysen,

c) Wahrscheinlichkeitsberechnungen (z.b.: Fehlerbaumanalyse),

d) Wirkung eines Ausfalls auf die Systemfunktionalität (z.b.: FMEA),

e) Instandhaltbarkeit (abgeleitet aus den Instandsetzungszeiten) basierend aus den Kenntnissen der Instandsetzungszeiten

\section{Qualitative/Quantitative Auswertungsmethoden}

Um die Funktionsweise und Aufgabenerfüllung von den produzierten Komponenten zu überprüfen, bedarf es an Methoden die Schwachstellen schon in der Entwicklung aufzeigen. Sie sollen helfen Ausfälle, Gefährdungen, Auswirkungen aber auch die Verbindungen untereinander zu identifizieren, um die Systeme auch demnach zu bewerten. Unterschieden werden zwischen (vgl.: Villemeur, 1992):

- Qualitativen Verfahren: Bewertung von Systemen erfolgt verbal oder über einfache Zahlenwerte. Ermittelt werden Wahrscheinlichkeiten von bestimmten Ereignissen. Weiter sind Qualitative Systemmodellierungen auch Voraussetzung für Quantitative Verfahren. Die am häufigsten Angewendeten Methoden sind in der Praxis die FMEA und die FTA. FMEA wird auch besonders in der Automobilindustrie angewendet. Besonderheit von FTA (Fault Tree Analysis) ist auch, dass sie Quantitativ angewendet werden kann (vgl. ebd.).

- Quantitative Verfahren: Ermittelt werden Ausfallwahrscheinlichkeiten mit Hilfe von Wahrscheinlichkeitsrechnungen. Es erfolgt eine statistische Bewertung eines Systems bzw. die Errechnung von Ausfallraten (vgl. ebd.).

Welches Verfahren bzw. Methode für Unternehmen am besten geeignet ist, hängt von der Zielsetzung und dem Systemumfang/Projektumfang ab. Nach Analyse der türkischen Zulieferer mit IRIS Zertifikat ist festzuhalten, dass in diesen Unternehmungen jedoch keine eigene Entwicklung von Produkten bzw. die Entwicklung und Design von eigenen Fahrzeugen erfolgt. Deshalb wird auch eine 


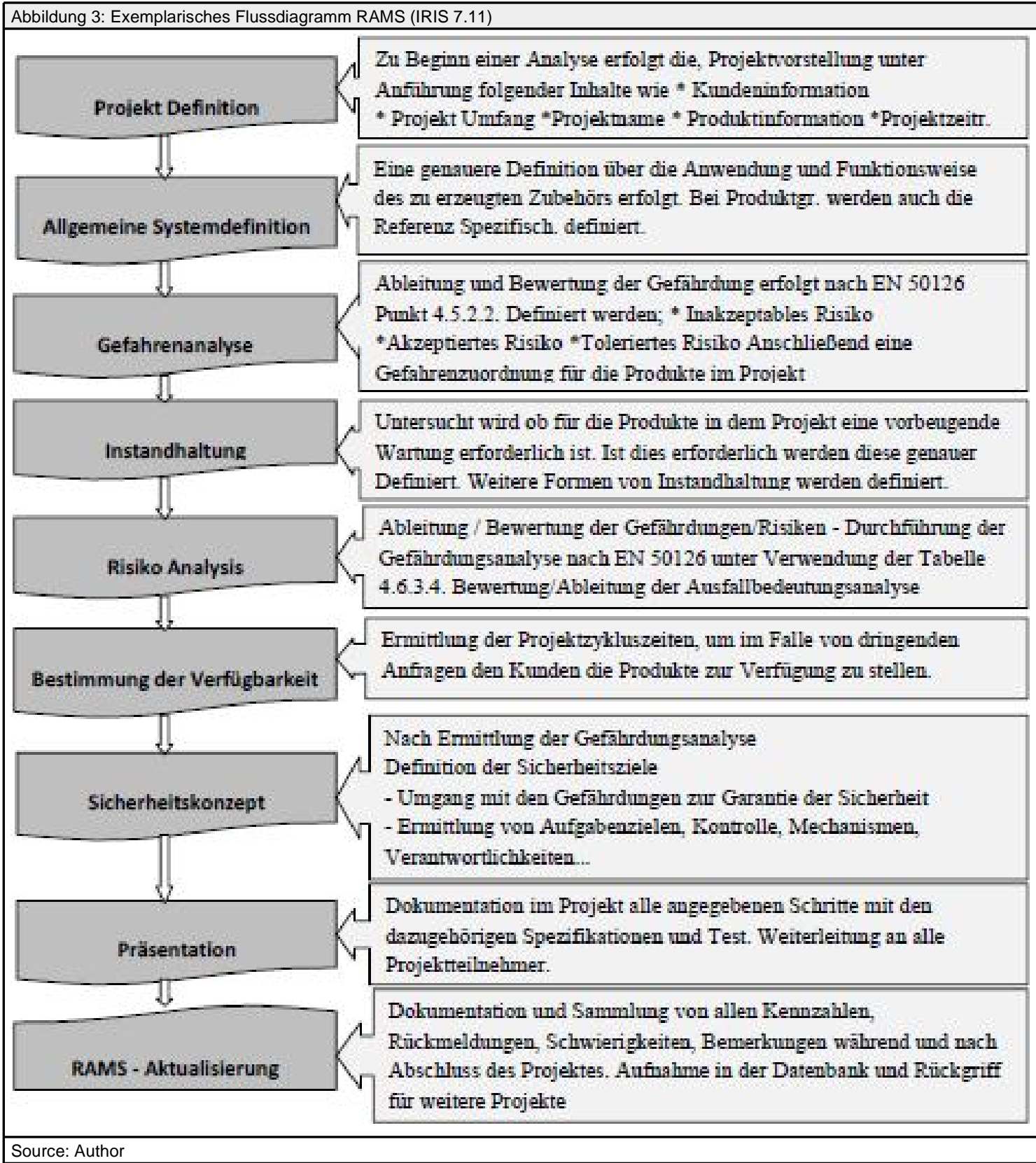

Umfangreiche Systemanalyse bzw. statische Analyse seitens dieser Unternehmen nicht benötigt. Nachdem auch viele dieser Unternehmen in der Automobilindustrie tätig sind, scheint vorerst eine FMEA Analyse auch für viele ausreichend zu sein.

\section{Exemplarisches RAMS Model}

Um den Unternehmen eine Hilfestellung in der Erarbeitung eines RAMS/LCC Models zugeben wurde ein exemplarisches Flussdiagramm ausgearbeitet, dass vor allem jenen Unternehmen die kein umfangreiches RAMS/LCC benötigen helfen soll die Mindestanforderung zu erfüllen. Nachdem das Unternehmen auch in der Automobilindustrie tätig ist, geht man bei der Erstellung des Flussdiagrammes von der Anwendung der FMEA Methode aus.
Das oben dargestellte Flussdiagramm ist nur eine Möglichkeit zur Erstellung eines Modells, weitere Variationen Schwerpunkte sind je nach Unternehmen möglich. Im Folgenden wird die im Flussdiagramm angeführte Punkt Risiko-Analyse genauer behandelt. Dabei wird die FMEA Methode verwendet.

\section{FMEA Prozess - Mögliche Anwendung in der Praxis}

FMEA ist eine qualitative Methode zur Ermittlung von möglichen Fehlern, Ausfällen und deren Ursachen sowie deren Folgen. Die Ergebnisse werden tabellarisch dargestellt die unter anderem Ausfallarten, Auswirkungen und anzuwendende Maßnahmen beinhalten (vgl. Barrantes, 2002, 74ff) 
Die FMEA kann in Bereichen wie Konstruktion oder Produktion oder in den verschiedenen Phasen des Produktentwicklungsprozesses eingesetzt werden (ebd.).

Als Exemplar wurde ein FMEA für ein Zubehör welches in der Schienenindustrie verwendet wird entwickelt. Aufgestellt wurde die FMEA für den Bereich der Montage in einem Unternehmen.

Ausgegangen wird von einem Bracket, welches vom Kunden nicht montiert werden kann. Definiert werden Art und Ursache für das Auftreten dieser Fehlermöglichkeit. Zur Risikobeurteilung werden die Wahrscheinlichkeit des Auftretens (A) des Fehlers ihre Folge bzw. Fehlerschwere (B) und ihre Entdeckungwahrscheinlichkeit (E) ermittelt und mit Hilfe der Risikoprioritätszahl RPZ bewertet (vgl. Kamiske, Brauer, 2006, 75)

$R P Z=A^{*} B^{*} E$

Für die Risikobeurteilung wird die Skala (Bewertungskatalog nach VDA 4.2) verwendet. Dabei gilt 1 als kein Risiko und 10 wird als hohes Risiko betrachtet, aus denen dann die Risikoprioritätszahl berechnet wird. Liegt die RPZ über einen vorgegebenen Wert sollten Verbesserungsmaßnahmen getätigt werden.

\section{Exemplarische Fehlerbaumanalyse (FTA) im Unternehmen}

Zur besseren Überschaubarkeit wird neben einer FMEA auch eine Fehlerbaumanalyse (FTA) durchgeführt. FTA

\begin{tabular}{|c|c|c|c|}
\hline \multicolumn{4}{|c|}{ Abbildung 4: Auszug aus dem Bewertungskatalog nach VDA 4.2} \\
\hline & \begin{tabular}{|l|} 
Bedeutung \\
Fehlerschwere B
\end{tabular} & $\begin{array}{l}\text { Auftretenswahr- } \\
\text { scheinlichkeit A }\end{array}$ & $\begin{array}{l}\text { Endeckungswahr } \\
\text {-scheinlichkeit E }\end{array}$ \\
\hline \begin{tabular}{|l|}
10 \\
9
\end{tabular} & Sehr hoch & \begin{tabular}{|l|} 
Sehr Hoch \\
$500.000-100.000 \mathrm{ppm}$
\end{tabular} & $\begin{array}{l}\text { Sehr gering } \\
90 \%\end{array}$ \\
\hline \begin{tabular}{|l|}
8 \\
7
\end{tabular} & Hoch & $\begin{array}{l}\text { Hoch } \\
50.000-10.000 \mathrm{ppm}\end{array}$ & $\begin{array}{l}\text { Gering } \\
98 \%\end{array}$ \\
\hline $\begin{array}{l}6 \\
5 \\
4\end{array}$ & Mittel & \begin{tabular}{|l|} 
Mittel \\
$5.000-1.000-500 \mathrm{ppm}$
\end{tabular} & \begin{tabular}{|l|} 
Mittel \\
$99.7 \%$
\end{tabular} \\
\hline$\frac{3}{2}$ & Gering & $\begin{array}{l}\text { Gering } \\
100-50 \mathrm{ppm}\end{array}$ & $\begin{array}{l}\text { Hoch } \\
99.9 \%\end{array}$ \\
\hline 1 & Sehr gering & $\begin{array}{l}\text { Sehr gering } \\
1 \mathrm{ppm}\end{array}$ & $\begin{array}{l}\text { Sehr Hoch } \\
99.99 \%\end{array}$ \\
\hline
\end{tabular}

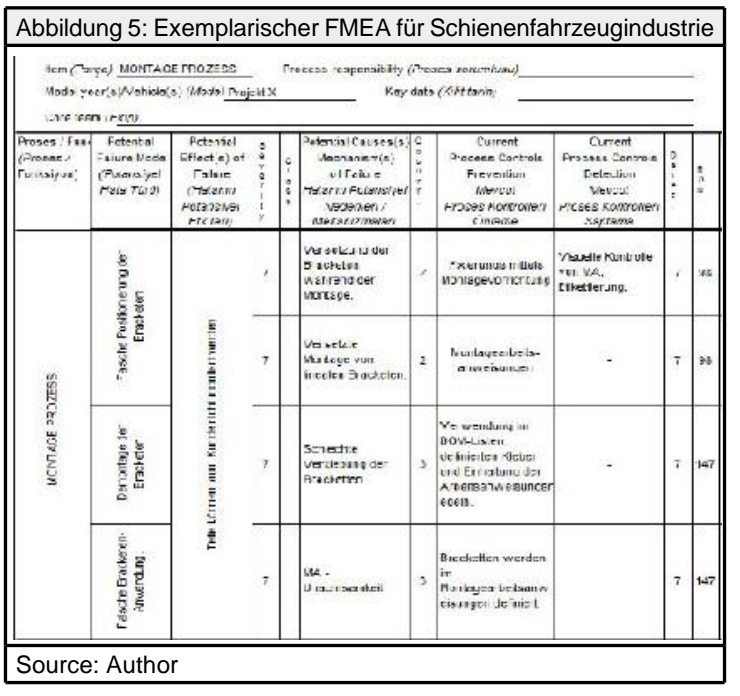

Methode wird bei der Identifizierung von Ursachen bestimmt, die zu unerwünschten Ereignisse (Events) durch und- oder- Verknüpfungen zum Top-Ereignis führen. Die Analyse Erfolgt Top-down daher vom Top-Ereignis zu den Ursachen. Für Ereignisse denen keine weiterer Ursache definiert werden können, werden als das Basis-Ereignis deklariert (Fendrich, 2007, 677ff) Die FTA bietet eine bessere Überschaubarkeit der Fehlerursachen.

Die Fehlerbaumanalyse kann sowohl qualitativ, als auch quantitativ erfolgen. Qualitativ zur Identifikation aller möglichen Ursachen des TOP-Ereignisses und Quantitativ zur Ermittlung der Eintrittswahrscheinlichkeit unerwünschter Ereignisse (ebd.).

Neben den erwähnten Vorteilen einer Fehlerbaumanalyse, ist ihre Anwendung bei komplexen Systemstrukturen mit hohem Aufwand verbunden und bedeutet auch gleichzeitig größere Fehlerbäume). Bei der Erstellung einer FTA ist eine genauere Kenntnis über das System, Ursachen, Kennzahlen usw. von großer Bedeutung, um alle möglichen Ursachen, Folgen auch genau mit einkalkulieren zu können. Auch ist zu bedenken, dass für jedes unerwünschte Ereignis ein eigener Fehlerbaum erstellt werden muss.

Begonnen wird mit dem Top Ereignis und Schritt für Schritt werden mögliche Ursachen für weitere Verknüpfungen bis zum Basis Ereignis aufgelistet. Dieses Beispiel ist exemplarisch $\mathrm{zu}$ sehen und ist auf jeden Fall Ausbaumöglich. Bei der Erstellung des Fehlerbaumes wurden die Daten von der FMEA verwendet.

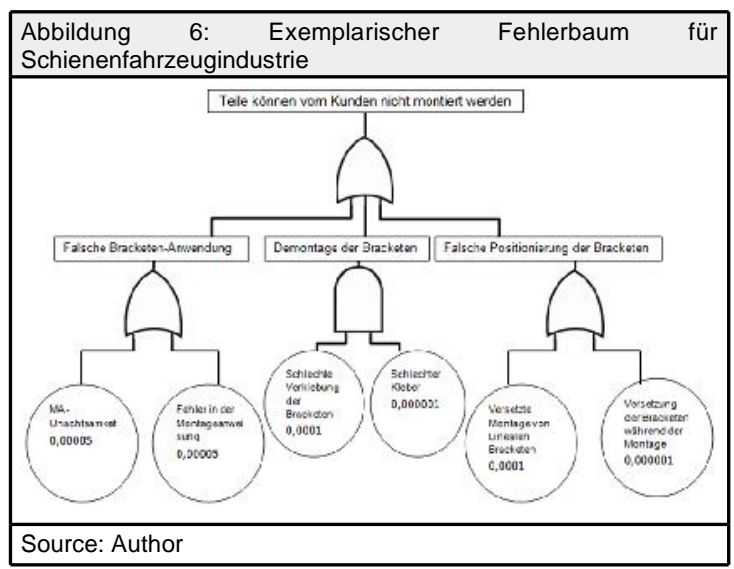

Sind die Wahrscheinlichkeiten der einzelnen Ereignisse bekannt und unabhängig voneinander, kann zusätzlich eine quantitative Auswertung erfolgen. Sobald mehr als die UNDbzw. ODER- Verknüpfungen enthalten sind, wird die Auswertung schwieriger.

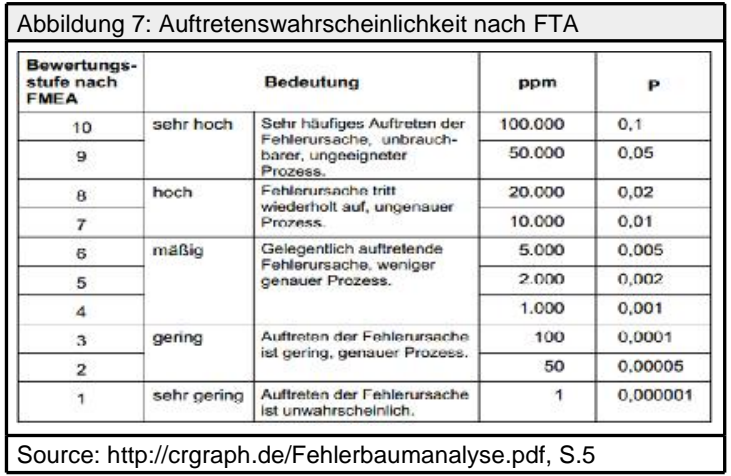


Risikobewertung für die Basis Ereignisse werden in Anlehnung an die FMEA Bewertungsstufe für Auftretenswahrscheinlichkeit entnommen.

FTA kann in den Unterschiedlichsten Entwicklungsphasen angewendet werden. Wichtig ist aber bei der Durchführung ein detailliertes Systemwissen, um alle möglichen und denkbaren Varianten zu identifizieren. FTA ist eine Graphische Darstellung der Fehlerkette in dem zu Beginn das TOP-Ereignis festgelegt wird und alle untergeordneten Ereignisse die dieses Ereignis Auslösen können, werden aufgelistet bist alle Ursachen daher das Basis - Ereignis gefunden wird. Da jedoch nicht immer alle Komponenten benannt werden, Fehler auch temporär auftreten können, kann diese durch sogenannte Platzhalter freigehalten werden, die dann später bestimmt werden können (vgl. ebd. ). Dies ist auch vor allem für die untersuchten Unternehmen von Vorteil, da wie schon erwähnt noch die Erfahrungen zu den einzelnen Komponenten fehlen.

Fazit

Resümierend ist zu sagen, dass IRIS ein Regelwerk ist, das trotz Schwierigkeiten in einigen Bereichen Zuspruch seitens der Unternehmer findet. Kritik ist im Bereich RAMS/LCC zu hören die sich aber vorwiegend aus unzureichende Wissensbestände in diesem Beriech zurückzuführen sind und nach dem erlernen dieses Handwerkes auch als überbrückbar scheint. RAMS Management ist sehr viel versprechend, doch überfordert es auch in seiner Komplexität Unternehmen aufgrund fehlender Kennzahlen, Erfahrungen und Analysemöglichkeiten.

Um eine Erleichterung als auch Hilfestellung zu leisten scheint für viele Unternehmen, nachdem sie auch schon in der Automobilindustrie tätig sind und mit Verfahren wie FMEA Erfahrungen aufweisen, vom Aufwand her fürs erste einer der geeignetsten Verfahren für die Ermittlung von Ausfallsraten.

Natürlich müssen auch die Unternehmen, im eigenen Ermessen darüber entscheiden, in wie weit sie auch Bereit sind weiter für zusätzliche Programme, Workshops zu investieren um auch ein besseren Überblick und bessere Daten zu erhalten. Denn langfristig Betrachtet ermöglich RAMS in vielerlei Hinsicht besseren Überblick über das Produkt, Produktionsaufwand und mit dem einhergehenden Kosten.

\section{REFERENCES}

Ahi, T. (2012). Die Lage der Eisenbahn in der Türkei 2012. In: Fern - Express: MARMARAY. Heft 4/2011.S 35-37.

Böhler, H. (2004). Marktforschung. Diller, H., Köhler, R.,(Hrgb.) Kohlhammer Edition Marketing. Auflage 3.

EN 50126 (1999).

EN ISO 9001:2008: Qualitätsmanagementsysteme Anforderungen

Früh, K.F., Maier, U., \& Schaudel, D. (2009). Handbuch der Prozessautomatisierung. Prozessleittechnik für verfahrenstechnische Anlagen. 4. Auflage, Oldebourg Industrieverlag GmbH. München, S746.

Hahn, A., \& Häusler, S. (2013). Quantitatives Entwicklungsmanagement. Modellbasierte Analyse von Produktentwicklungsprozessen. Springer Vieweg, Oldenburg. International Railway Industry Standard (2009).

Kamiske, G. -F., \& Brauer, J. -P. (2006): Qualitätsmanagement von $A$ bis $Z$, Verlag Hanser München, $S 75$.

Osanna, P. H., Durakpasa, M. N., \& Afjehi-Sadat, A.(2004) Qualitätsmanagement und Prüfwesen, Abteilung für Austauschbau und Messtechnik, Grafisch Zentrum Verlag, Wien.
Petrellis, E., \& Schmitz, B. (2010). QM-Systeme im EmbeddedMarkt von strategischer Bedeutung. IN. RDV/TÜV Media GmbH (Hrsg.): Management und Qualität. Das Magazin für integrierte Managementsysteme. Ausgabe Deutschland.

Pfitzinger, E. (2009). Projekt DIN EN ISO 9001:2008: Vorgehensmodell zur Implementierung eines Qualitätsmanagementsystems. Beuth Verlag. Berlin.

Rötzel, A., \& Rötzel-Schwunk, (1999). Qualitätsmanagement Massgeschneidert- für kleinere und mittlere Betriebe, VDE- Verlag, Berlin.

UNIFE The European Railway industry (2009). IRIS International Railway Industry Standard. Deutsch Revision 02.

Villemeur, A. (1992). Reliability, availability, maintainability and safety assessment - Volume 1: Methods and Techniques. John Wiley and Sons Ltd, Chichester, ISBN 0-471-93048-2.

http://www.crgraph.de/Fehlerbaumanalyse.pdf, S.5 